\title{
BMJ Open A three-year follow-up on the efficacy of psychosocial interventions for patients with mild dementia and their caregivers: the multicentre, rater-blinded, randomised Danish Alzheimer Intervention Study (DAISY)
}

\author{
Kieu T T Phung, ${ }^{1}$ F B Waldorff, ${ }^{1,2}$ D V Buss, ${ }^{1}$ A Eckermann, ${ }^{1}$ N Keiding, ${ }^{3}$ \\ S Rishøj, ${ }^{1}$ V Siersma, ${ }^{2}$ J Sørensen, ${ }^{4}$ R Søgaard, ${ }^{4}$ L V Sørensen, ${ }^{1}$ A Vogel, ${ }^{1}$ \\ G Waldemar ${ }^{1}$
}

To cite: Phung KTT, Waldorff FB, Buss DV, et al. A three-year follow-up on the efficacy of psychosocial interventions for patients with mild dementia and their caregivers: the multicentre, rater-blinded, randomised Danish Alzheimer Intervention Study (DAISY). BMJ Open 2013;3:e003584. doi:10.1136/bmjopen-2013003584

- Prepublication history and additional material for this paper is available online. To view these files please visit the journal online (http://dx.doi.org/10.1136/ bmjopen-2013-003584).

Received 11 July 2013 Revised 12 October 2013 Accepted 15 October 2013

CrossMark

For numbered affiliations see end of article.

Correspondence to Dr Kieu T T Phung; thien.phung@mail.mcgill.ca

\section{ABSTRACT}

Objectives: To examine the long-term efficacy at the 36-month follow-up of an early psychosocial counselling and support programme lasting 8-12 months for community-dwelling patients with mild Alzheimer's disease and their caregivers.

Design: Multicentre, randomised, controlled, raterblinded trial.

Setting: Primary care and memory clinics in five Danish districts.

Participants: 330 home-dwelling patients with mild Alzheimer's disease and their primary caregivers (dyads)

Interventions: Dyads were randomised to receive intervention during the first year after diagnosis. Both intervention and control groups had follow-up visits at $3,6,12$ and 36 months.

Main outcome measures: Primary outcomes for the patients assessed at 36-month follow-up were changes from baseline in global cognitive function (Mini-Mental State Examination), depressive symptoms (Cornell Depression Scale) and proxy-rated EuroQoL quality of life on visual analogue scale. The primary outcomes for the caregivers were changes from baseline in depressive symptoms (Geriatric Depression Scale) and self-rated EuroQoL quality of life on a visual analogue scale. The secondary outcome measures for the patient were proxy-rated Quality of Life Scale for Alzheimer's disease (QoL-AD), Neuropsychiatric InventoryQuestionnaire, Alzheimer's disease Cooperative Study Activities of Daily Living Scale, all-cause mortality and nursing home placement.

Results: At a 36-month follow-up, 2 years after the completion of the Danish Alzheimer Intervention Study (DAISY), the unadjusted positive effects previously detected at the 12-month follow-up in one patient primary outcome (Cornell depression score) and one patient secondary outcome (proxy-rated QoL-AD) disappeared (Cornell depression score, $p=0.93$; proxyrated QoL-AD, $p=0.81$ ). No long-term effect of DAISY

\section{Strengths and limitations of this study}

- This is the largest randomised controlled trial of early psychosocial intervention for patients with mild Alzheimer' disease and their caregivers to date, with a long follow-up of 3 years.

- It is a study of solid methodology, strictly adhering to CONSORT recommendations.

- The multicomponent semitailored intervention programme was intensive in both content and duration, targeted multiple needs, tended to the individual needs and simultaneously involved caregivers and patients; thus, having the characteristics that defined successful intervention programmes documented in the literature.

- Multiple primary and secondary outcomes were chosen based on the specific aims of DAISY and on the outcomes from similar intervention studies for patients with more advanced dementia. To avoid finding spurious effects, a conservative significance level was set at $p=0.0005$.

- All patients had primary caregivers who are very much involved in caregiving, a situation that cannot be generalised to all patients with dementia in Denmark.

- There was assessment of need at baseline.

- Intervention lasted 1 year but without continuous follow-up and support during the subsequent 2 years.

intervention on any other primary and secondary outcomes was found at the 36-month follow-up.

Conclusions: For patients with very mild Alzheimer's disease and their caregivers, an intensive, multicomponent, semitailored psychosocial intervention programme with counselling, education and support during the first year after diagnosis did not show any positive long-term effect on primary and secondary outcomes. 
Trial registration: The study was registered in the Clinical Trial Database (http://www.controlled-trials.com/ISRCTN74848736).

\section{INTRODUCTION}

Psychosocial interventions for patients with Alzheimer's disease (AD) and their caregivers have gained recognition during the past two decades. The majority of patients with dementia live in their own homes with their caregivers, usually their spouses, who bear the responsibility of caregiving. ${ }^{1}$ Caring for family members with dementia has long been considered as the most stressful type of family caregiving, predisposing caregivers to mental and physical illnesses and increasing their risk for death. ${ }^{2}$ Previously an under-researched area, the needs of patients with $\mathrm{AD}$ have received more attention in recent years, with studies documenting their need for information about their illness, for help to cope with their disabilities, for social recognition and support and for a decent quality of life with meaningful social contact and activities. ${ }^{3}$ Patients' unmet needs can result in mood and behavioural problems, safety issues, social isolation and increased risk for nursing home placement and death. ${ }^{4}$ Meta-analyses and systematic reviews of the numerous clinical trials assessing the efficacy of psychosocial interventions for caregivers have shown a significant effect of interventions on reducing caregivers' psychological morbidity and reduce patients' neuropsychiatric symptoms. ${ }^{5-9}$ Studies examining the effect of psychosocial intervention on patients' mortality and nursing home placement are scarce and the results are inconsistent. ${ }^{5}{ }^{10}$ Studies that included psychosocial interventions for the patients are limited, providing anecdotal evidence for positive effects of interventions on patients' cognitive function, psychological morbidity and time to nursing home placement. ${ }^{10}$ Today, thanks to the remarkable advances in diagnosing dementia, patients can be diagnosed at an early stage when their relatively intact autonomy and insight enable them to convey their needs and actively participate in intervention programmes. The rapidly growing number of people with $\mathrm{AD}$ in the coming years, a considerable proportion of them diagnosed in the early stages, presents a pressing need to develop and validate intervention programmes that focus on the needs of patients with mild dementia and their caregivers and involve both parties in the intervention.

It was hypothesised that the Danish Alzheimer Intervention Study (DAISY), a multifaceted and semitailored intervention programme offered to patients with $\mathrm{AD}$ and their primary caregivers during the first year after the diagnosis, could have a long-term effect in preventing the emergence of depressive symptoms, improving quality of life for the patients and the caregivers, stabilising the patients' cognitive function and delaying nursing home placement. ${ }^{1}$ The results of the 12-month follow-up were published in $B M J$ in 2012, showing no significant difference in outcomes between the DAISY and the control groups. ${ }^{11}$ However, the significant level corrected for multiple testing $(\mathrm{p}=0.0005)$ was subsequently criticised for being too conservative, given that an alternative correction method could have given another conclusion. ${ }^{12}$ Before adjustment for multiple testing was carried out, the data analysis of the results at the 12-month follow-up had shown statistical significance in one primary patient outcome (Cornell Depression Scale score, $\mathrm{p}=0.0103$ ) and one secondary patient outcome ( proxy-rated quality of life QoL-AD, $\mathrm{p}=0.0013$ ) in favour of the DAISY group. ${ }^{11}$ Therefore, a 36-month follow-up was subsequently carried out to follow the evolution of these outcomes. This paper reports the results of this follow-up.

\section{METHODS}

Detailed description of the study rationale, methods, design, randomisation and sample size has been published. ${ }^{1}$

\section{Trial design}

DAISY was a large multicentre, rater-blinded, 1-year randomised controlled trial of the efficacy of intensive psychosocial intervention for patients with mild $\mathrm{AD}$ and their caregivers. It was an exploratory randomised clinical trial with multiple primary and secondary outcomes.

\section{Participants}

The patients were recruited from five Danish districts. One designated memory clinic in each district recruited the patients for the trial. Each recruiting centre had one study co-ordinator and one physician who assessed the patients for eligibility. Patients were referred from local memory clinics as well as private practice in psychiatry, neurology, geriatrics and family medicine. If referred from private practice, dementia diagnosis was confirmed by specialists in the recruiting memory clinic.

The inclusion criteria were (1) home-living patients diagnosed within the past 12 months with $\mathrm{AD}$, mixed $\mathrm{AD}$ with vascular component or Lewy body dementia; (2) 50 years of age or older; (3) Mini-Mental State Examination (MMSE) score $\geq 20^{13}$ and (4) having one participating primary caregiver. The primary caregiver was defined as the main person responsible for the informal care of the patient with minimum weekly contact. All patients met DSM-IV criteria for dementia, ${ }^{14}$ NINCDS-ADRDA criteria for probable $\mathrm{AD}^{15}$ or McKeith criteria for Lewy body dementia. ${ }^{16}$ Patients with mixed $\mathrm{AD}$ were those with probable $\mathrm{AD}$ and minor vascular changes on cranial CT that could contribute to their symptoms.

Patients with severe somatic or psychiatric comorbidities (including impaired hearing or vision) that would significantly impair their compliance with the DAISY programme were excluded. Patients who had already 
been involved in other intervention programmes were also excluded. Patient-caregiver dyads were randomised to the DAISY group, in which they were provided with intensive psychosocial interventions and follow-up support at 3, 6 and 12 months; or to the control group, in which they were only provided with follow-up support at 3, 6 and 12 months. The study was subsequently extended and the patients and their caregivers were asked to give a separate consent to an additional follow-up at 36 months.

\section{Intervention}

A multifaceted and semitailored psychosocial intervention programme described in details in our previous reports ${ }^{11}$ was designed to provide counselling, information and support to patients with mild dementia and their caregivers in the intervention group. The study co-ordinator in each centre, an experienced nurse specialising in caring for patients with dementia and having received special training in counselling for the study (constructivist approach) ${ }^{17}$ implemented the intervention within the first month after inclusion in the trial. Consisting of five key components, the intervention focused on positive resources, intact function, retained skills and feasible activities for the patients: (1) the study co-ordinator provided seven individual counselling sessions tailored to the needs of the patients and their caregivers: two for the patient alone, two for the caregiver alone, two for the patient-caregiver dyad and one with the dyad together with their family network (optional); (2) the study co-ordinator provided outreach telephone counselling 5-8 times with 3-4 week intervals to maintain regular contact and follow-up on the individual counselling sessions; (3) using log books, the patients and their caregivers independently kept track of the thoughts and daily issues that they wanted to discuss at the counselling sessions; (4) experts in the field of dementia were invited to teach five standard courses as group intervention with separate courses for patients and caregivers to provide general information about dementia and forum for discussion, sharing information and support and (5) patients and caregivers were provided with information folders produced especially for the purpose of the study about dementia causes, diagnosis and treatment, legal issues and resources for social support. The intervention programme lasted 8-12 months. Full compliance was defined as adherence with the major components of the intervention programme: patients who participated with their caregivers in at least three counselling sessions (not including the optional network session) and in at least three teaching course sessions.

The patients in both the intervention and the control groups were followed up at 3, 6, 12 and 36 months. Attempts were made to provide similar treatment for both intervention and control participants in all respects other than the add-on DAISY. At each follow-up visit, participants in both groups were interviewed about their current symptoms and daily-life issues, and informed about available support programme (if any) in their local communities. Both groups were free to participate in such support programmes during the study and participation in these support activities was registered for both groups. Identified special needs led to referral to local care facilities when available and relevant.

\section{Outcomes}

\section{Primary outcomes for the patients}

1. Global cognitive function: The patient's global cognitive function was assessed using MMSE. ${ }^{13}$ The sum of scores ranges from 0 to 30 . Higher scores indicate better cognitive function.

2. Depressive symptoms: Cornell Scale for Depression in Dementia was used to assess the patient's depressive symptoms through an interview with both the patient and caregiver. ${ }^{18}$ The scale has 19 items, each item rating a specific depressive symptom in increasing severity (0-2), yielding a total score ranging from 0 to 38 , with higher scores indicating more depressive symptoms. A score $\geq 8$ indicates significant depressive symptoms and a score $\geq 10$ indicates major depression.

3. Proxy-rated quality of life: The primary caregiver evaluated the patient's health-related quality of life using the EuroQoL EQ-5D, ${ }^{19}$ a questionnaire inquiring about mobility, self-care, activities, pain, discomfort, anxiety and depression. Quality of life was rated using a visual analogue scale (EQ-VAS) with scores ranging from 0 to 100 . Higher scores signify better quality of life.

\section{Primary outcomes for the primary caregivers}

1. Depressive symptoms: The caregivers rated their own depressive symptoms using the Geriatric Depression Scale. ${ }^{20}$ The total score ranges from 0 to 30 with higher score indicating more depressive symptoms. A cut-off score of 10 distinguishes between depressed and non-depressed individuals.

2. Self-rated quality of life: The caregivers rated their own health-related quality of life using the EQ-VAS. ${ }^{19}$ The scores range from 0 to 100 with higher scores indicating good quality of life.

\section{Secondary outcomes for the patients}

1. Proxy-rated quality of life, AD-specific: The caregiver rated the patient's quality of life using Quality of Life Scale for Alzheimer's Disease (QoL-AD), ${ }^{21}$ a 13 -item scale measuring disease-specific quality of life in people with $\mathrm{AD}$. Total score ranges from 13 to 52 with higher scores indicating better quality of life.

2. Neuropsychiatric symptoms: The patient's neuropsychiatric and behavioural symptoms were assessed through an interview with the caregiver using Neuropsychiatric Inventory-Questionnaire. ${ }^{22}$ Total score ranges from 0 to 36 with higher scores indicating more severe disturbances. 
3. Activities of daily living: The caregiver completed the Alzheimer's Disease Cooperative Study Activities of Daily Living Scales for clinical trials in Alzheimer's disease (ADCS-ADL) ${ }^{23}$ to assess the patient's activities of daily living. ADCS-ADL is a 23-item scale with total scores ranging from 0 to 78 . Higher scores indicate better functioning.

4. Mortality and nursing home placement: The Danish Civil Registration System ${ }^{24}$ was used together with personal contact with the caregivers to collect information regarding death and nursing home placement. In case of doubt, the local district authority or the residential place was contacted to check whether the address was registered as a nursing home.

\section{BASELINE AND FOLLOW-UP ASSESSMENTS}

Both patients and their caregivers were invited to participate in all the assessments. The local study coordinator carried out the baseline assessment prior to randomisation at the local study centre. Independent raters blind to group assignment carried out follow-up assessments at 6, 12 and 36 months during home visits. The raters were neither involved in the intervention programme nor employed in the same institutions as the study co-ordinators. The efficiency of concealment was checked through questionnaires administered to the raters at the end of each follow-up visit. None of the raters visited the same patient-caregiver dyad more than once.

\section{Statistical methods}

Characteristics and outcome measures at baseline of the dyads in the intervention and control groups were compared using Student $t$ tests for continuous variables and $\chi^{2}$ tests for categorical variables. With linear models on the full data of up to four observations per dyad, we compared the difference in development of the primary and secondary outcomes between randomisation groups during the follow-up period, using generalised estimating equations to account for repeated measurements; the inclusion of a categorical centre indicator variable account for possible clustering by treating centre. To adjust for possible bias because of differential death and dropout from the study between the intervention and control groups, the assessments at the various follow-up times were weighted by the inverse of an estimate of the probability of staying in the study, a method explained in the seminal paper by Dufouil et $a l^{25}$ These probabilities were estimated from the data in logistic regression models for death and dropout with the dyads' characteristics and the observed primary outcomes from previous visits as covariates. Only the expected scores and inferences for the 36-month follow-up were reported. Differences in mortality and nursing home placement rates between the two groups were evaluated by HR from a Cox regression model. All analyses were carried out using the intention-to-treat principle.

\section{RESULTS}

Five hundred and fifty-eight patients were screened for eligibility and 330 patient-caregiver dyads were included: 163 were randomised to DAISY group and 167 to control group (figure 1). Their demographics, clinical characteristics and outcome measures at baseline are provided in table 1. Most patients received cognition-enhancing medications $(93.3 \%$ cholinesterase inhibitor and $1 \%$ NMDA receptor antagonist). ${ }^{1}$ Overall, the participation rate in the DAISY group was high. ${ }^{11}$

At 36 months, a total of 130 patients (67 in the intervention group and 63 in the control group) were lost for follow-up (figure 1). In all, 56 patients had deceased, 36 from the DAISY group and 20 from the control group (figure 1). Patients in the DAISY had a higher mortality rate (HR 1.99; 95\% CI 1.15 to 3.43; $\mathrm{p}=0.01$ ). Regarding nursing home placement, 43 patients from DAISY group and 48 from the control group were placed in nursing homes at the 36-month follow-up. Data on nursing home placement was missing for five participants in the intervention group. There was no difference between the rates of nursing home placement for the intervention and control groups (HR $0.97 ; 95 \%$ CI 0.64 to $1.47 ; \mathrm{p}=0.89$ ).

As reported previously, the 12-month follow-up study observed positive effects of DAISY on preventing the emergence of depressive symptoms (Cornell depression scale, primary patient outcome) and maintaining quality of life (proxy-rated QoL-AD, secondary patient outcome). ${ }^{11}$ The effect size of DAISY regarding Cornell depression score was -1.58 ( -2.79 to $-0.37, \mathrm{p}=0.0103$ ) and regarding proxy-rated QoL-AD was 2.14 (0.83 to 3.45; $\mathrm{p}=0.0013$ ). In this 36-month follow-up study, which took place after DAISY had stopped for 2 years, there was no significant difference between intervention and control groups regarding these two outcomes (Cornell depression score, $\mathrm{p}=0.93$; proxy-rated $\mathrm{QoL}-\mathrm{AD}, \mathrm{p}=0.82$; tables 2 and 3). The effect size of DAISY regarding Cornell depression score was $-0.06 \quad(-1.43$ to 1.32 ; $\mathrm{p}=0.93)$ and regarding proxy-rated QoL-AD was -0.19 $(-1.75$ to $1.38, \mathrm{p}=0.82)$. Furthermore, the 36-month follow-up study did not find any long-term effect of DAISY on any of the other primary and secondary outcomes (tables 2 and 3).

At baseline, the patients were at the very early stage of dementia with a mean MMSE of 24.1 (SD 2.6). At the 36-month follow-up, there was a marked fall in MMSE mean scores of 6-7 in both groups, accompanied by a marked deterioration in the patients' quality of life (table 2). Additionally, the patients were well functioning in their ADL and had very few behavioural problems at baseline. At the 36-month follow-up, ADL had deteriorated markedly and behavioural symptoms had emerged (table 2). Participants in both group had few depressive symptoms at baseline and minimal changes in their mean Cornell Depression Scale scores at the 36-month follow-up as compared to the baseline (table 2 and 3 ).

The study found no effect of DAISY on caregivers' selfrated quality of life and depressive symptoms at the 

Alzheimer Intervention Study.

(1) Patients and caregivers.

(2) Full compliance is defined as participation from both the caregiver and the patient in at least three courses and three counselling sessions each. (3) In the analysis accounting for dropouts, information from all participating dyads were incorporated.
Figure 1 Trial flow for Danish

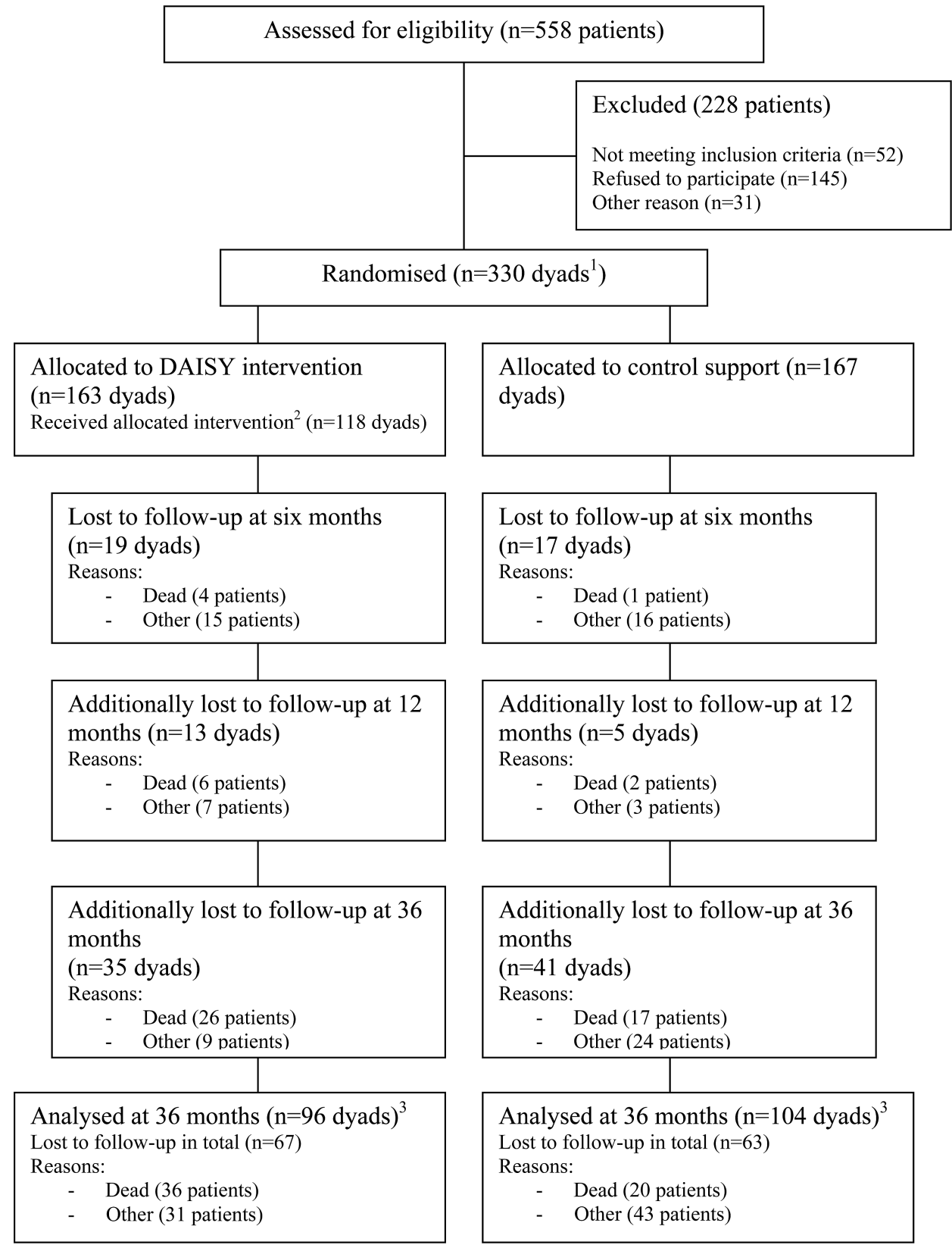

36-month follow-ups. The caregivers were characterised by a lack of depressive symptoms and a high self-rated quality of life at the baseline (table 1). At the 36-month follow-up, their depressive symptoms and self-rated quality of life had changed minimally from baseline (tables 2 and 3).

\section{DISCUSSION}

This study did not find any long-term effect of an intensive psychosocial intervention (DAISY) on patients and caregivers beyond the effect of structured follow-up support.

To our knowledge, this study is the largest randomised controlled trial of early psychosocial intervention for patients with mild $\mathrm{AD}$ and their caregivers to date, with a long follow-up of 3 years. It is a study of solid methodology, strictly adhering to CONSORT recommendations. A priori sample size calculation was carried out. The measures for primary and secondary outcomes are reliable scales, which are commonly used in routine clinical practice and in intervention studies across cultures. $^{26} \quad 27$ Proper randomisation, allocation concealment, rater-blinded evaluation of outcomes and adjustment for multiple testing were rigorously carried out to reduce biases that could lead to type I errors. ${ }^{11}$ The multicomponent semitailored intervention programme was intensive in both content and duration, targeted multiple needs, tended to the individual needs and simultaneously involved caregivers and patients; thus, having the characteristics that defined successful intervention programmes documented in the literature. $^{2} 57$ Since ours was one of the first studies to examine the effect of support and counselling 
Table 1 Baseline characteristics of patients with Alzheimer's disease and their caregivers who participated in the Danish Alzheimer Intervention Study (DAISY)

Intervention $(n=163)$

Control $(n=167)$

\section{Patients' characteristics}

Sex

Male

Female

Mean (SD) age (years)

Household status

Living alone

Living with others

Home

Rented

Owned

$76(46.6)$

$87(53.4)$

$76.5(7.7)$

$92(55.1)$

$75.9(6.6)$

54 (33.1)

109 (66.9)

48 (28.7)

$119(71.3)$

Education

66 (40.5)

97 (59.5)

56 (33.5)

$111(66.5)$

None

60 (36.8)

39 (23.9)

$<3$ years

64 (39.3)

$57(34.1)$

49 (29.3)

$\geq 3$ years

$61(36.5)$

Charlson comorbidity index

64 (39.3)

$75(46.0)$

$24(14.7)$

73 (43.7)

1 comorbidity

$65(38.9)$

$\geq 2$ comorbidities

$29(17.4)$

Diagnosis

Pure Alzheimer's disease

$112(68.7)$

$44(27.0)$

7 (4.3)

$127(76.1)$

38 (22.8)

2 (1.2)

Caregiver's characteristics

Sex

Male

$54(33.1)$

$109(66.9)$

65.5 (12.7)

56 (33.5)

Female

Mean (SD) age (years)

$104(63.8)$

45(27.6)

14 (8.6)

$111(66.5)$

Relation

Spouse

Child or child in law

Other

Living with patient

Yes

No

$101 / 162$ (62.4)

61/162 (37.6)

$66.5(12.7)$

$111(66.5)$

$41(24.5)$

$15(9.0)$

Home

Rent

45 (27.6)

$118(72.4)$

$112 / 166(67.5)$

$54 / 166$ (32.5)

Own

41 (25.2)

46 (28.2)

76 (46.6)

45 (26.9)

$122(73.1)$

None

$<3$ years

$\geq 3$ years

$37 / 166$ (22.3)

$63 / 166(37.9)$

$66 / 166$ (39.8)

Outcome measures at baseline

Primary patient outcomes

Mean (SD) MMSE

Mean (SD) Cornell Depression Scale

$24.0(2.5)$

$24.1(2.7)$

$5.2(4.8)$

$4.4(4.0)$

$62.1(18.4)(n=162)$

$64.7(20.4)$

Primary caregiver outcome

Mean (SD) EQ-VAS

79.3 (16.3) $(n=162)$

$81.4(16.3)$

Mean (SD) GDS

$4.74(5.2)(n=162)$

$4.71(5.0)$

Secondary patient outcome Mean (SD) QoL-AD (proxy-rated)

$33.0(6.1)$

$34.7(6.6)$

3.9 (3.6)

$3.9(3.7)$

Mean (SD) NPI-Q
Mean (SD) ADSC-ADL

$61.2(11.4)$

$61.8(11.4)$

Values are numbers (percentages) of participants unless stated otherwise.

ADSC-ADL, Alzheimer's Disease Cooperative Study Activities of Daily Living Scale; EQ-VAS, European Quality of Life Visual Analogue Scale; GDS, Geriatric Depression Scale; MMSE, Mini-Mental State Examination; NPI-Q, Neuropsychiatric Inventory Questionnaire; QoL-AD, Quality of Life Scale for Alzheimer's Disease. 
Table 2 Outcome measures of the DAISY psychosocial interventions based on completed response at 36-month follow-up

\begin{tabular}{|c|c|c|c|c|}
\hline & \multicolumn{2}{|l|}{ Observed scores } & \multicolumn{2}{|c|}{ Changes from baseline } \\
\hline & Intervention & Control & Intervention & Control \\
\hline \multicolumn{5}{|l|}{ Primary patient outcomes } \\
\hline MMSE & $17.8(6.7)(\mathrm{n}=84)$ & $17.9(7.1)(n=94)$ & $-6.21(6.17)(n=84)$ & $-6.35(6.26)(n=94)$ \\
\hline Cornell Depression Scale & $5.57(4.78)(n=93)$ & $5.17(4.19)(n=101)$ & $1.29(4.94)(n=93)$ & $0.74(4.45)(n=101)$ \\
\hline Proxy-rated EQ-VAS & $50.7(20.3)(n=95)$ & $52.3(21.0)(n=102)$ & $-12.88(20.3)(n=95)$ & $-12.46(19.0)(n=102)$ \\
\hline \multicolumn{5}{|l|}{ Primary caregiver outcomes } \\
\hline EQ-VAS & $79.4(16.1)(n=94)$ & $79.0(18.0)(n=103)$ & $-0.79(16.5)(n=94)$ & $-1.49(16.5)(n=103)$ \\
\hline GDS & $5.26(5.43)(n=94)$ & $4.51(5.26)(n=103)$ & $0.81(4.83)(n=94)$ & $0.14(4.52)(n=103)$ \\
\hline \multicolumn{5}{|l|}{ Secondary patient outcomes } \\
\hline QoL-AD (proxy-rated) & $30.5(5.1)(n=96)$ & $32.1(6.2)(n=103)$ & $-2.89(4.89)(n=96)$ & $-2.84(2.00)(n=103)$ \\
\hline NPI-Q & $5.21(4.43)(n=96)$ & $5.05(4.80)(n=104)$ & $1.57(4.43)(n=96)$ & $1.20(4.68)(n=104)$ \\
\hline ADSC-ADL & $35.3(19.4)(n=96)$ & $41.3(20.8)(n=104)$ & $-26.7(16.6)(n=96)$ & $-22.3(19.6)(n=104)$ \\
\hline
\end{tabular}

Values are means (SD) unless stated otherwise.

ADSC-ADL, Alzheimer's Disease Cooperative Study Activities of Daily Living Scale; DAISY, Danish Alzheimer Intervention Study; EQ-VAS, European Quality of Life Visual Analogue Scale; GDS, Geriatric Depression Scale; MMSE, Mini Mental State Examination; NPI-Q,

Neuropsychiatric Inventory Questionnaire; QoL-AD, Quality of Life Scale for Alzheimer's Disease.

programmes in patients with very mild dementia, no previous consensus exists concerning gold standards for assessing efficacy. Therefore, multiple primary and secondary outcomes were exploratively chosen based on the specific aims of the DAISY and on the outcomes from similar intervention studies for patients with more advanced dementia. ${ }^{1}$ Consequently, to avoid finding spurious effects, a significance level was set at $\mathrm{p}=0.0005$, which was subsequently criticised for being too conservative. ${ }^{12}$ All patients in this study had primary caregivers who were very involved in caregiving, a situation that cannot be generalised to all patients with dementia in Denmark.

Although not statistically significant for this adjusted $p$ value, DAISY did produce small positive effects on reducing depressive symptoms and maintaining quality of life for the patients at the 12-month follow-up. ${ }^{11}$ The effect size of DAISY regarding Cornell depression score was 1.58 (-2.79 to $-0.37, \mathrm{p}=0.0103)$ and regarding proxyrated QoL-AD was 2.14 (0.83 to 3.45; $\mathrm{p}=0.0013)$. The disease-specific QoL-AD is probably more sensitive to measure the effect of psychological interventions than general EQ-VAS. ${ }^{27}$ At the 36-month follow-up, these positive effects were no longer present. Between 12 and 36 months of follow-up, there was significant decline in patients' cognition, quality of life and ADL. During this time period, there was no continuing intervention or support. Initially, the study was intended to end at the 12-month follow-up. However, we received additional funding to carry out follow-up at 36 months. The timing and duration of DAISY could have missed a period of significant decline when intervention could have been more beneficial. Possibly, the positive trends observed at the 12-month follow-up could have been maintained or enhanced had the intervention continued for an additional 2 years. Evidence from the very limited literature seems to support the hypothesis that the positive effects of psychosocial interventions could be lost without continuous reinforcement. There are few randomised controlled trials assessing the efficacy of psychosocial intervention that specifically targets community-dwelling patients with dementia. ${ }^{6}{ }^{10}$ Most trials had short follow-up period, usually 3-6 months. One trial showed that a 3-month programme of intensive physical exercise for the patients combined with teaching caregivers strategies to manage patients' behavioural problems improved the patients' physical functioning and depressive symptoms. ${ }^{28}$ At 24-month follow-up, the improvement in physical functioning was still significant, but the improvement of depressive symptoms was no longer present. ${ }^{28}$ In contrast, another trial with 8-year follow-up reported delayed nursing home placement for patients by providing a multicomponent interventions for the caregivers and patients; the 10-day intervention programme was followed by continuous support over the telephone, weekly for the first year and yearly thereafter for the next 7 years. $^{29}$

In our study, there are some possible explanations for the non-significant positive effects found at the 12-month follow-up concerning patients' depressive symptoms and quality of life and the disappearance of these effects at the 36-month follow-up. First, it could be a floor effect. Our patients had minimal depressive symptoms and relatively high scores of QoL-AD at baseline. A randomised controlled trial using support group intervention for community-dwelling patients with mild $\mathrm{AD}$ and their caregivers showed that patients who experienced improvement in their depressive symptoms had significantly more depressive symptoms at baseline and a higher level of distress. ${ }^{30}$ Second, there was no need assessment at baseline. Probably, participants with more symptoms and at greater need should have received the full intensive intervention programme and regular support follow-up was sufficient for those who had minimal symptoms and needs at baseline. Third, the control group also received some intervention that is 


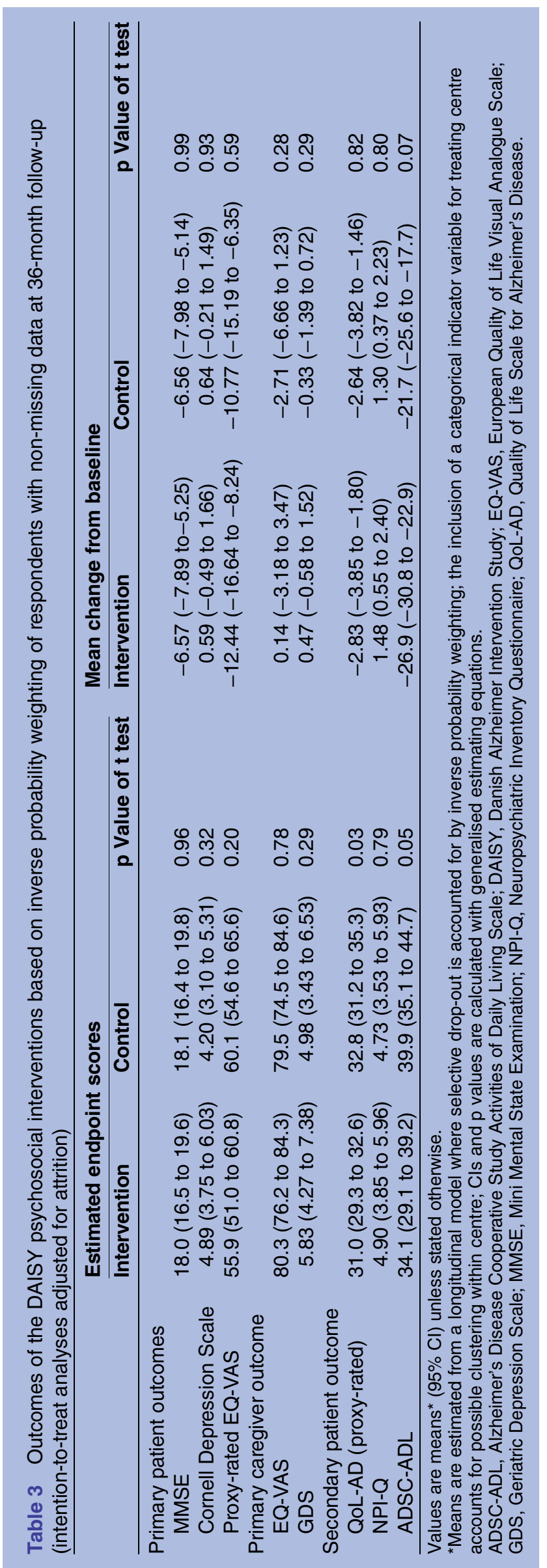

much better than the usual practice in Denmark. ${ }^{31}$ They had regular follow-ups when they could speak about emerging psychosocial and health problems, receive information about available resources, and get referred to relevant health professionals if needed. It is noteworthy that despite the marked decline in patients' global cognitive function, quality of life and ADL between 12 and 36 months of follow-ups, participants in both groups had minimal changes in mean Cornell Depression Scale scores as compared to the baseline. This could be an indication that the regular follow-ups offered in this study were sufficient enough to produce a long-term effect in preventing the conversion into clinical depression for the patients. Fourth, as aforementioned, the intervention should probably continue longitudinally following the clinical progression in these patients to show long-term positive effects. The study did not find any long-term effect on DAISY on the caregiver outcomes. Previous studies have shown positive responses to interventions from caregivers with high levels of depression and anxiety at baseline. ${ }^{10}$ For this mostly asymptomatic group of caregivers in our study, perhaps follow-up at regular intervals provided enough information and support to prevent the emergence of depressive symptoms and maintain good life quality.

It is not known why patients in the intervention group had higher mortality than those in the control group. This increased mortality was unlikely to be caused by the intervention, as the nature of the intervention programme did not subject the patients to any health risk. Using the data from Statistics Denmark (http://www.dst. $\mathrm{dk}$ ), the incidence of death for the age-matched general population over the same time period was found to be similar to that of the DAISY group. The control group, however, had lower incidence of death compared with the general population. At baseline, the quality of life of the patients in the intervention group was rated as poorer than that of the control group, both by the patients themselves and by their caregivers (table 1). Although not statistically significant, there were small socioeconomic and clinical differences that could be responsible for the higher mortality rate in the intervention groups. More patients in the intervention group lived alone (4\% difference), rented their house ( $7 \%$ difference), had more comorbidities (4.4\% difference) and were diagnosed with mixed $\mathrm{AD}$ and vascular dementia $(4.2 \%$ difference, table 1$)$. Whether these differences could contribute to the higher mortality in the intervention group is uncertain. It is known that older people living alone have higher mortality than those living with others. ${ }^{32}$ Currently, there is insufficient evidence in the literature concerning the effect of psychosocial intervention on patient mortality, as studies looking at this effect are scarce. $^{53}$

The same patient characteristics in the DAISY group stated above could also explain the lack of effect concerning nursing home placement. ${ }^{34}$ Additionally, continuous intervention and follow-up between 12 and 
36 months could have been needed to produce a positive long-term effect on nursing home placement. Randomised controlled trials that reported positive longterm effect of psychosocial intervention on patients' nursing home placement provided continuous support and counselling over the phone for $8-9$ years. ${ }^{29} 35$ In contrast, intervention lasting 2 years but without continuous follow-up and support showed no long-term effect on nursing home placement. ${ }^{33}$

Although this study found no long-term effect of DAISY, a qualitative study linked to this randomised controlled trial showed that $80 \%$ of patients and $94 \%$ of caregivers in the intervention group found the intervention overall beneficial. Patients felt that their self-esteem was improved and they could better manage their daily life and social relations. Caregivers felt that they were more confident and competent to cope with the challenges of caring for relatives with $\mathrm{AD}$. After the intervention, patients and caregivers looked for support groups to join permanently and caregivers sought continued counselling. ${ }^{36}$ In contrast to randomised clinical trials about pharmacological interventions, we did not carry out DAISY to justify the reason for providing psychosocial intervention for patients with dementia and their caregivers, whose need for information, counselling and support cannot be denied. What we can conclude from this study is that since we could not show positive effects in the quantitative analyses, we should not offer psychosocial intervention indiscriminately to all patients with very mild dementia and their caregivers, but we should probably assess their needs and offer intervention only to those who need it. Regular follow-up is therefore important to identify the arising needs that require intervention. Probably, the type, dose and intensity, and duration of early intervention should be more tailored to match the needs of patients and their caregivers at baseline to maximise benefit, economise resources and avoid unnecessary intervention burden. The intervention programme should perhaps be designed in such a way that patients and caregivers with greater needs at baseline receive more intensive interventions that cater to their specific needs, those with lesser needs receive a basic intervention programme of lower intensity and those with minimal or no needs receive no intervention at all. To obtain long-term effect, early intervention should probably have a longitudinal and fluid course that follows the disease progression, being continuously modified according to the needs that arise. These are the questions to be answered in future studies.

\footnotetext{
Author affiliations

${ }^{1}$ Memory Disorders Research Group, Danish Dementia Research Centre, Department of Neurology, Copenhagen University Hospital Rigshospitalet, Copenhagen, Denmark

${ }^{2}$ Research Unit and Department of General Practice, Institute of Public Health, University of Copenhagen, Copenhagen, Denmark

${ }^{3}$ Department of Biostatistics, Institute of Public Health, University of Copenhagen, Copenhagen, Denmark

${ }^{4}$ Centre for Applied Health Services Research (CAST), University of Southern Denmark, Odense, Denmark
}

Acknowledgements The authors would like to thank 128 volunteers who acted as raters and assistants from the 68 municipalities and five counties and the following project co-ordinators and physicians in the five participating centres for their contributions to patient recruitment, intervention and data collection: Ribe County: Esbjerg Hospital, Department of Psychiatry (Anna Marie Hansen, Johanne Christensen, Jørgen Jensen). Ringkoebing County: Herning Hospital, Department of Psychiatry (Marianne Refslund, Birgitte Aagaard, Palle Lund) and Holstebro Hospital, Department of Psychiatry (Inge Lund Petersen, Finn Andersen). Roskilde County: Roskilde Hospital, Department of Geriatrics (Dorte Dyre, Lisbeth Petersen, Birgitte Froelund, Lise Korbo, Ellen Holm) and Department of Neurology (Kurt Luedorff $\mathbf{t}$ ). Vestsjaelland County: Korsoer Hospital, Department of Geriatrics (Mette Lassen, Lars Laugesen) and Dianalund hospital, Department of Psychiatry (Thyge Jensen, Ole Bjoern Skausig). Copenhagen Capital area: Hvidovre Hospital, Department of Geriatrics (Lillian Moerk Joergensen), Amager Hospital, Department of Geriatrics (Suzanne Sanders), Bispebjerg Hospital, Department of Geriatrics (Claus Moe $\dagger$ ), Frederiksberg Community Health Care Center (Ingrid Lauridsen), Frederiksberg Hospital, Department of Psychiatry (Rene Klysner), Glostrup Hospital, Department of Neurology, (Jens Feilberg), Rigshospitalet, Department of Neurology (Anne Eckerman, Eva Illemann, Peter Johannsen).

Contributors KTTP drafted the manuscript. FBW, VS, NK, MLHR and GW outlined the statistical analysis. VS conducted the statistical analysis in consultation with NK. FBW, AE, DVB, AV and GW designed and conducted DAISY. KTTP, FBW and GW worked on interpretation of data analysis. All authors assisted in editing this manuscript. All authors read and approved the final manuscript. All authors are guarantors for the scientific integrity of the article.

Funding DAISY was supported by the National Board of Social Services at the Danish Ministry of Social Affairs (grant number J.nr. 8742010024), the Danish Ministry of Health (grant number J.nr. 2003-11031-5) and the Danish Health Foundation (grant number J.nr. 2002D070, 2004D220 and 2005D228).

Competing interests GW had support from the National Board of Social Services at the Danish Ministry of Social Affairs, the Danish Ministry of Health and the Danish Health Foundation, for the submitted work.

Ethics approval The Danish Data Protection Agency approved the research database (ID No 2003-41-3178). All patients and caregivers gave informed consent to participate in the trial and the extended 36-month follow-up.

Provenance and peer review Not commissioned; externally peer reviewed.

Data sharing statement The dataset is available on request to Gunhild Waldemar (Gunhild.waldemar@rh.regionh.dk)

Open Access This is an Open Access article distributed in accordance with the Creative Commons Attribution Non Commercial (CC BY-NC 3.0) license, which permits others to distribute, remix, adapt, build upon this work noncommercially, and license their derivative works on different terms, provided the original work is properly cited and the use is non-commercial. See: http:// creativecommons.org/licenses/by-nc/3.0/

\section{REFERENCES}

1. Waldemar G, Waldorff FB, Buss DV, et al. The Danish Alzheimer intervention study: rationale, study design and baseline characteristics of the cohort. Neuroepidemiology 2011;36:52-61.

2. Schulz R, Martire LM. Family caregiving of persons with dementia: prevalence, health effects, and support strategies. Am J Geriatr Psychiatry 2004;12:240-9. Review.

3. van der Roest HG, Meiland FJ, Maroccini R, et al. Subjective needs of people with dementia: a review of the literature. Int Psychogeriatr 2007;19:559-92.

4. Gaugler JE, Kane RL, Kane RA, et al. Unmet care needs and key outcomes in dementia. J Am Geriatr Soc 2005;53:2098-105.

5. Brodaty $\mathrm{H}$, Green A, Koschera A. Meta-analysis of psychosocial interventions for caregivers of people with dementia. J Am Geriatr Soc 2003;51:657-64.

6. Brodaty $\mathrm{H}$, Arasaratnam $\mathrm{C}$. Meta-analysis of nonpharmacological interventions for neuropsychiatric symptoms of dementia. Am J Psychiatry 2012;169:946-53. 
7. Pinquart M, Sorensen S. Helping caregivers of persons with dementia: which interventions work and how large are their effects? Int Psychogeriatr 2006;18:577-95.

8. Thompson CA, Spilsbury K, Hall J, et al. Systematic review of information and support interventions for caregivers of people with dementia. BMC Geriatr 2007;7:18.

9. Schoenmakers B, Buntinx F, DeLepeleire J. Supporting the dementia family caregiver: the effect of home care intervention on general well-being. Aging Ment Health 2010;14:44-56. Review.

10. Olazarán J, Reisberg B, Clare L, et al. Nonpharmacological therapies in Alzheimer's disease: a systematic review of efficacy. Dement Geriatr Cogn Disord 2010;30:161-78.

11. Waldorf FB, Buss DV, Eckermann A, et al. Efficacy of psychosocial intervention in patients with mild Alzheimer's disease: the multicentre, rater blinded, randomised Danish Alzheimer Intervention Study (DAISY). BMJ 2012;345:e4693.

12. Mittelman MS. The DAISY psychosocial intervention does not improve outcomes in patients with mild Alzheimer's disease or their carers. Evid Based Ment Health 2013;16:15.

13. Folstein MF, Folstein SE, McHugh PR. 'Mini-mental State'. J Psychiat Res 1975;12:189-98.

14. American Psychiatric Association (APA). Diagnostic and statistical manual of mental disorders. DSM-IV, 4th edn. Washington DC: American Psychiatric Association, 1993.

15. McKhann $G$, Drachman D, Folstein $M$, et al. Clinial diagnosis of Alzheimer's disease: report of the NINCDS-ADRDA Work Group under the auspices of Department of Health and Human Services Task Force on Alzheimer's Disease. Neurology 1984;34:939-44.

16. McKeith IG, Galasko D, Kosaka K, et al. Consensus guidelines for the clinical and pathological diagnosis of dementia with Lewy bodies (DLB): report of the consortium on DLB international workshop. Neurology 1996;47:1113-24.

17. Peavey VR. Sociodynamic counseling: a constructivist perspective for the practice of counselling in the 21st century. Victoria: Trafford Publishing, 1997.

18. Alexopoulos GS, Abrams RC, Young RC, et al. Cornell scale for depression in dementia. Biol Psychiatry 1988;23:271-84.

19. The EuroQoL Group. EuroQoL-a new facility for the measurement of health-related quality of life. Health Policy 1990;1:199-208.

20. Yesavage JA, Brink TL, Rose TL, et al. Development and validation of a geriatric depression screening scale: a preliminary report. $J$ Psychiatr Res 1982;17:37-49.

21. Logsdon RG, Gibbons LE, McCurry S, et al. Quality of life in Alzheimer's disease: patient and caregiver report (QoL-AD). J Ment Health Aging 1999;5:21-32.

22. Kaufer DI, Cummings JL, Ketchel P, et al. Validation of the NPI-Q, a brief clinical form of the neuropsychiatric inventory. J Neuropsychiatry Neurosci 2000;12:233-9.
23. Galasko $D$, Bennett $D$, Sano $M$, et al. An inventory to assess activities of daily living for clinical trials in Alzheimer's disease. The Alzheimer's Disease Cooperative Study. Alzheimer Dis Ass Dis 1997;11(Suppl 2):S33-9.

24. Pedersen CB, Gøtzsche H, Møller JØ, et al. The Danish Civil Registration System. Dan Med Bull 2006;53:441-9.

25. Dufouil C, Brayne C, Clayton D. Analysis of longitudinal studies with death and drop-out: a case study. Stat Med 2004;23:2215-26.

26. Verhey FR, Houx P, Van Lang N, et al. Cross-national comparison and validation of the Alzheimer's Disease Assessment Scale: results from the European Harmonization Project for Instruments in Dementia (EURO-HARPID). Int J Geriatr Psychiatry 2004; 19:41-50

27. Moniz-Cook E, Vernooij-Dassen M, Woods R, et al:; INTERDEM group. A European consensus on outcome measures for psychosocial intervention research in dementia care. Aging Ment Health 2008;12:14-29.

28. Teri L, Gibbons LE, McCurry SM, et al. Exercise plus behavioral management in patients with Alzheimer disease: a randomized controlled trial. JAMA 2003;290:2015-22.

29. Brodaty H, Gresham M, Luscombe G. The Prince Henry Hospital dementia caregivers' training programme. Int J Geriatr Psychiatry 1997;12:183-92.

30. Logsdon RG, Pike KC, McCurry SM, et al. Early-stage memory loss support groups: outcomes from a randomized controlled clinical trial. J Gerontol B Psychol Sci Soc Sci 2010;65:691-7.

31. Phung TK, Andersen BB, Kessing LV, et al. Diagnostic evaluation of dementia in the secondary health care sector. Dement Geriatr Cogn Disord 2009;27:534-42

32. Lund R, Due P, Modvig J, et al. Cohabitation and marital status as predictors of mortality-an eight year follow-up study. Soc Sci Med 2002;55:673-9.

33. Brodaty $\mathrm{H}$, Mittelman M, Gibson $\mathrm{L}$, et al. The effects of counselling spouse caregivers of people with Alzheimer disease taking donepezil and of country of residence on rates of admission to nursing homes and mortality. Am J Geriatr Psychiatry 2009;17:734-43.

34. Wattmo C, Wallin AK, Londos E, et al. Risk factors for nursing home placement in Alzheimer's disease: a longitudinal study of cognition, ADL, service utilization, and cholinesterase inhibitor treatment. Gerontologist 2011;51:17-27.

35. Mittelman MS, Haley WE, Clay OJ, et al. Improving caregiver well-being delays nursing home placement of patients with Alzheimer disease. Neurology 2006;67:1592-9.

36. Sørensen LV, Waldorff FB, Waldemar G. Early counselling and support for patient with mild Alzheimer's disease and their caregivers. A qualitative study on outcome. Aging Ment Health 2008;12:444-50. 